\title{
Mitigation of ionospheric effects on GNSS
}

\author{
René Warnant $\left({ }^{1}\right)$, Ulrich Foelsche $\left({ }^{2}\right)$, Marcio Aquino $\left({ }^{3}\right)$, Benoit Bidaine $\left({ }^{4}\right)$, Vadim Gherm $\left({ }^{9}\right)$, \\ Mohammed Mainul Hoque $\left({ }^{8}\right)$, Ivan Kutiev $\left({ }^{5}\right)$, Sandrine Lejeune $\left({ }^{1}\right)$, Juha-Pekka Luntama $\left({ }^{6}\right)$, \\ Justine Spits $\left({ }^{1}\right)$, Hal J. Strangeways $\left({ }^{7}\right)$, Gilles Wautelet $\left({ }^{1}\right)$, Nikolay Zernov $\left({ }^{9}\right)$ and Norbert Jakowski $\left({ }^{8}\right)$ \\ $\left(^{1}\right)$ Royal Meteorological Institute, Brussels, Belgium \\ (') WegCenter and IGAM, University of Graz, Austria \\ $\left(^{3}\right)$ IESSG, University of Nottingham, UK \\ $\left(^{4}\right)$ University of Liège, Belgium \\ $\left(^{5}\right)$ Geophysical Institute, Bulgarian Academy of Sciences (BAS), Sofia, Bulgaria \\ $\left(^{6}\right)$ Finnish Meteorological Institute, Helsinki, Finland \\ $\left.{ }^{7}\right)$ School of Electronic and Electrical Engineering, University of Leeds, UK \\ ${ }^{8}$ ) Deutsches Zentrum für Luft- und Raumfahrt (DLR), Neustrelitz, Germany \\ ( $\left.{ }^{9}\right)$ Department of Radio Physics, University of St. Petersburg, Petrodvorets, Russia
}

\begin{abstract}
The effects of the ionosphere remain one of the main factors which limit the precision and the reliability of many GNSS applications. It is therefore indispensable on the one hand to improve existing mitigation techniques and on the other hand to assess their remaining weaknesses. Mitigation techniques depend on the type of application considered. Therefore, specific mitigation techniques have to be developed. The paper summarizes work performed on this topic in the frame of WP 3.2 «Mitigation techniques» of COST 296.
\end{abstract}

Key words ionosphere - mitigation - TEC - GNSS - positioning - radio-occultation

\section{Introduction}

Space based radio systems like Global Navigation Satellite Systems (GNSS) are affected by the effects of the ionosphere. Nowadays, GNSS are used to measure positions in the frame of many different types of applications.

Mailing address: Dr. René Warnant, Royal Meteorological Institute, Avenue Circulaire 3, B-1180 Brussels, Belgium; e-mail: Rene.Warnant@ulg.ac.be
The obtained position accuracy which ranges from a few $\mathrm{mm}$ to about a decameter depends on the type of observables (code or phase measurements), the positioning mode (absolute or differential) or the fact that positions are computed in real time or in post-processing. GNSS are not only used for positioning applications. Indeed, GNSS are now recognized tools for atmospheric studies: for example, GNSS radiooccultation experiments allow reconstructing vertical electron density and temperature profiles in the atmosphere. The effects of the ionosphere remain one of the main factors which limit the precision of many applications. It is therefore indispensable to improve existing mitigation techniques. The Total Electron Content is the key parameter for the mitigation of the ionospheric error. Nevertheless, the way the ionosphere influences GNSS data processing 
techniques depends very much on the type of application. Therefore, specific mitigation techniques have to be developed. Ionospheric effects are also an important limitation to the reliability of GNSS applications: when mitigation techniques fail, the «nominal» precision level is not reached; in this case, users are not necessarily aware about the problem. This is an important issue for European positioning system Galileo which is supposed to give certified precision levels to its customers. This is particularly true for so-called «Safety of Life» applications like landings of air planes. Therefore, it is also important to assess remaining weaknesses in mitigation techniques.

The paper reviews work performed in the frame of Work Package 3.2 «Mitigation techniques» of COST Action 296 to improve ionosphere mitigation techniques in the frame of different applications and to assess their remaining weaknesses. At the present time, a growing number of users need results in real time. Such applications are particularly vulnerable to ionospheric threats. Therefore, we focus on real time ionospheric corrections.

\section{Real time ionospheric corrections for Single frequency users using NeQuick model}

Single frequency GNSS receivers constitute the simplest and most common systems which need real time mitigation for ionospheric effects.

To this extent they take benefit from quickrun empirical models such as NeQuick chosen in the framework of the European positioning system Galileo (Orus et al., 2007). This global model provides monthly median electron density profiles for given time, location and solar flux (Radicella and Leitinger, 2001). It is therefore well suited for Total Electron Content (TEC) computation thanks to electron density integration along satellite-to-receiver ray paths.

This parameter is indeed needed for the mitigation of the ionospheric delay affecting GNSS applications and handled for Galileo by the so-called Single Frequency Ionospheric Correction Algorithm. NeQuick is based on monthly median maps - known as CCIR maps - of ionosonde parameters. Consequently the Galileo algorithm includes the computation of an effective ionization level replacing solar flux in order to adapt the model to actual conditions.

Since the definition of this algorithm, a new version of NeQuick - called v2 from now on - has been released (Nava et al., 2008). It involves simplifications in the representation of the bottomside as well as a unique formula for a key parameter of the topside formulation previously defined through two equations, each one used for six months of the year. At the University of Liège, to compare both versions of the model to actual data, we initially

Table I. Comparison between NeQuick version 1 and 2 and GPS vertical TEC in 2002.

\begin{tabular}{ccccc}
\hline \hline & v1 & v2 & Evolution \\
\hline TECmeas [TECu] & & 23.2 & & \\
TECmod [TECu] & 21.7 & 20.9 & $-3.7 \%$ \\
Bias [TECu] & 1.5 & 2.3 & $52.1 \%$ \\
Relative [\%] & 6.6 & 10.0 & \\
RMS [TECu] & 7.0 & 4.5 & $-35.3 \%$ \\
Relative [\%] & 30.0 & 19.4 & \\
\hline
\end{tabular}



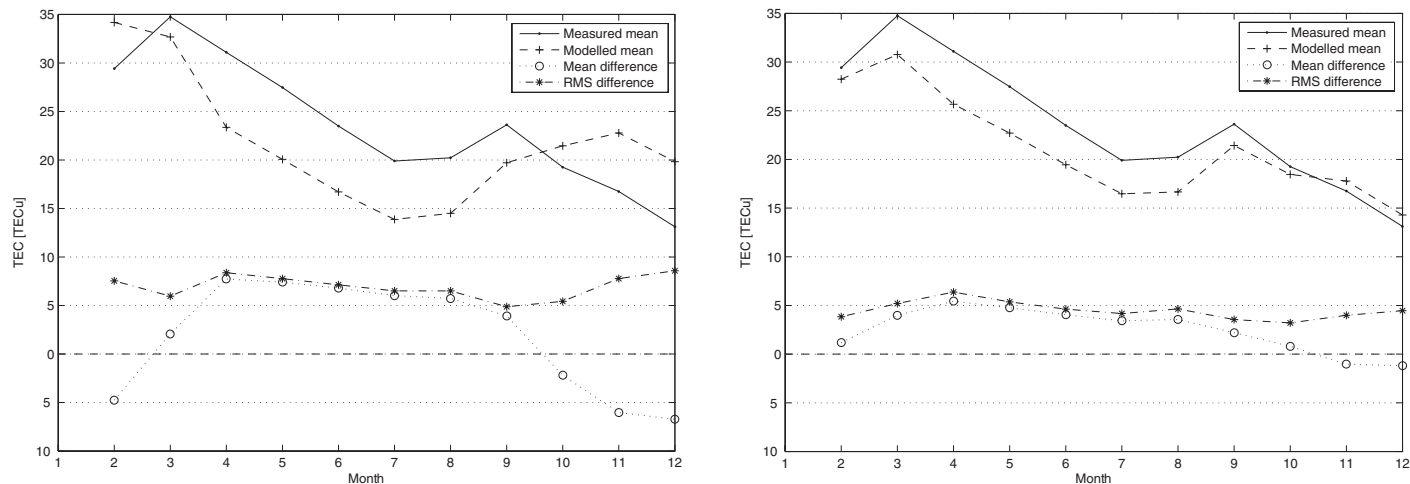

Fig. 1. Comparison between NeQuick version 1 (left) and 2 (right) and GPS vertical TEC in 2002: monthly statistics.

chose one analysis method which allows to uncouple NeQuick formulation from its underlying data i.e. the CCIR maps, replacing them by digisonde measurements. Then we computed vertical TEC and compared it with GPS TEC.

We performed tests for mid-latitudes (Dourbes Geophysical Observatory in Belgium) and high solar activity level (2002) using hourly data points for the whole year with a $77 \%$ availability level (Bidaine and Warnant, 2007). We obtained the global (yearly) statistics of table I showing an average underestimation of NeQuick TEC, slightly worse for the second version. However we observe a major improvement from NeQuick v2 as the Root Mean Square (RMS) difference decreases by more than a third.

We attribute this evolution mainly to the unification of the topside parameter formula as the six-month inversion of the monthly mean difference visible for NeQuick v1 has nearly completely disappeared in the second version (fig. 1).

Further investigations highlighted remaining weaknesses in the topside and we are currently analysing results for other stations and years.

The final goal of our study is to try to improve the Galileo single frequency algorithm results among others.

\section{Real time ionospheric corrections based on operational GNSS measurements}

\subsection{Real time corrections using ground based measurements}

The use of ground based observations of the satellite transmission to perform 2-D ionospheric tomography was first proposed in Austen et al. (1988). Continuous visibility and stability of the transmitted signals make GNSS satellites ideal for this purpose. Ground based GNSS observation networks provide a continuous flow of information that can be used for real time monitoring of the characteristics of the ionosphere and its dynamic processes. However, sophisticated data processing techniques are needed to fully exploit the potential of the GNSS observations. As a return, real time ionospheric monitoring can potentially allow regional mitigation of the ionospheric errors in GNSS applications with a very good accuracy and in real time.

Prerequisites for high resolution real time ionospheric tomography are timely availability of the observation data and sufficiently dense networks of observing stations. At the moment the large open GNSS networks like IGS (International GNSS Service), SuomiNet and EPN (EUREF Permanent Network) provide a very good starting point for ionospheric tomography. 


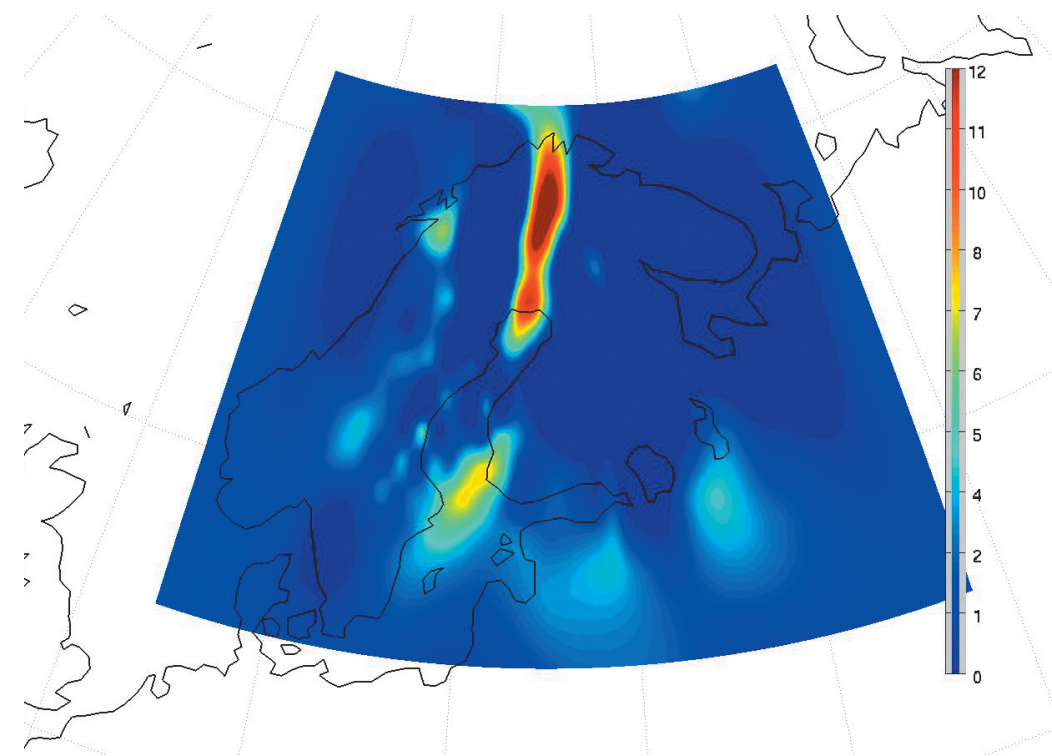

Fig. 2. A regional TEC map retrieved from slant GPS observations with the MIDAS algorithm. The colour bar shows the TEC values in TECU $\left(1 \mathrm{TECU}=10^{16}\right.$ electrons $\left./ \mathrm{m}^{2}\right)$.

The constraints of these networks are the sparse observations especially at high latitude regions and delayed availability of the data. The latter constraint is not critical for scientific research, but it would be a significant problem in the development of an operational GNSS error mitigation service. Regional networks often provide data with less delay and from a denser distribution of stations than global networks. Especially commercial GNSS networks are typically designed for real time services to assist satellite navigation applications. Dense regional GNSS networks are also used to provide information on crustal deformation related to seismic activity (e.g. California and Japan). Use of regional GNSS networks for ionospheric tomography has been demonstrated e.g. by Lee et al. (2007), Ma et al. (2005) and Wen et al. (2008).

FMI (Finnish Meteorological Institute) has assessed the feasibility of using observations from a regional GNSS network in Finland to perform mesoscale ionospheric tomography in the Auroral region. The auroral region is challenging due to the complexity of the ionosphere, especially during geomagnetic storms. The observation system used in this work is the VRS (Virtual Reference Station) network in Finland operated by Geotrim Ltd. This network contains 86 GNSS ground stations providing two frequency GPS and GLONASS observations. The sampling rate of the observations is $1 \mathrm{~Hz}$ and all observation data is transferred to the central processing facility in near real time (NRT).

The software used by FMI for ionospheric tomography is the MIDAS (Multi-Instrument Data Analysis System) algorithm developed and implemented by the University of Bath (Mitchell and Spencer, 2003). MIDAS is an extension into 3-D of the 2-D tomography algorithm originally presented by Fremouw et al. (1992). The MIDAS algorithm is based on a linear least squares inversion that can fit relative GPS differential phase data to corresponding data formed from integrations through an orthogonal basis set of model ionospheres. Electron density and TEC (Total Electron Content) maps retrieved with MIDAS have been used in many studies (e.g. Meggs et al. (2004) Mate- 
rassi and Mitchell (2005); Meggs et al. (2005); Yin et al. (2004); Yin and Mitchell (2005)).

The research performed at FMI in the framework of the COST 296 action has been based on observation data set collected from the Geotrim network in December 2006. This proved to be very good test period as at least two geomagnetic storms took place during the month. The periods of disturbed ionosphere in combination with the very quiet winter days of low solar activity form a good basis for assessing the performance of the tomography algorithm.

Figure 2 shows an example of a vertical TEC map at 02:45 UTC during the geomagnetic storm on 15 December 2006. The TEC map has been integrated from a 3-D electron density map retrieved from slant GPS observations. These results are very promising as for the first time the retrieved maps seem to capture the mesoscale features of the ionospheric plasma. However, validation of the high resolution electron density and TEC maps is a challenge as independent reference observations with a similar resolution are not available. The validation plans include intercomparison of the retrieved ionospheric characteristics with observations from ground based magnetometer and auroral camera network (MIRACLE), riometers and ionosonde station at Sodankylä Geophysical Observatory (SGO). The most promising reference for ionospheric tomography is the Ionospheric Tomography Chain operated by SGO. This chain provides $2-\mathrm{D}$ electron density plots in a vertical plane above the chain of five receivers located in Finland and Sweden.

The high resolution 3-D electron density maps can be used to estimate the ionospheric refractivity error for any GNSS receiver within the region covered by the retrieved solution e.g. in mobile single frequency GNSS applications the ionospheric correction can be determined based on the rough location of the receiver. This type of service requires production of ionospheric error corrections in real time. This requires regional nowcasting of the ionospheric characteristics. The main bottle neck at the moment in the development of an operational service is the coverage of the available observations. Using just one network seriously limits the coverage of the retrieved electron density maps. EUMETNET (The Network of European Meteorological Services) has set up the EGVAP (EUMETNET GPS water vapour programme) project to collect NRT observations from European GNSS stations. The current EGVAP network covers more than 400 GNSS sites. Collaboration with the E-GVAP project could be a potential way for collecting observations for a Europe-wide NRT ionospheric monitoring and nowcasting service.

\subsection{Real time corrections using ground and space based measurements}

The permanent monitoring of the structure and dynamics of the ionosphere is a key to successfully overcoming problems associated with the ionospheric impact on GNSS applications including both regular effects as well as perturbation induced signal degradation. DLR Neustrelitz has established a near real time ionospheric data service using three types of GNSS measurements illustrated in fig. 3. (http://w3swaci.dlr.de).

Dual frequency GPS measurements over the European area are used to derive the integral of the electron density (TEC) between the transmitting GNSS satellite and the receiver. Considering the increasing availability of dense GPS networks and the fact that one receiver may track up to more 10 satellites simultaneously, a large number of measurements can be obtained by ground based measurements. Before the TEC measurements can really be used, the hardware biases of individual satellite-receiver pairs have to be removed by calibration procedures based on Kalman filtering. After calibrating the linkrelated TEC data the observations are assimilated into an ionospheric model for creating TEC maps. The European TEC maps are updated every 5 minutes making them attractive for single frequency users to correct remaining ionospheric errors. The spatial grid resolution is $1 \mathrm{x} 1$ deg. These data are used to support GPS reference networks or single point positioning. As pointed out in Section 7 TEC data can also be used to correct higher order effects (Jakowski et al., 2005a,b,c; 2006; 2007). 


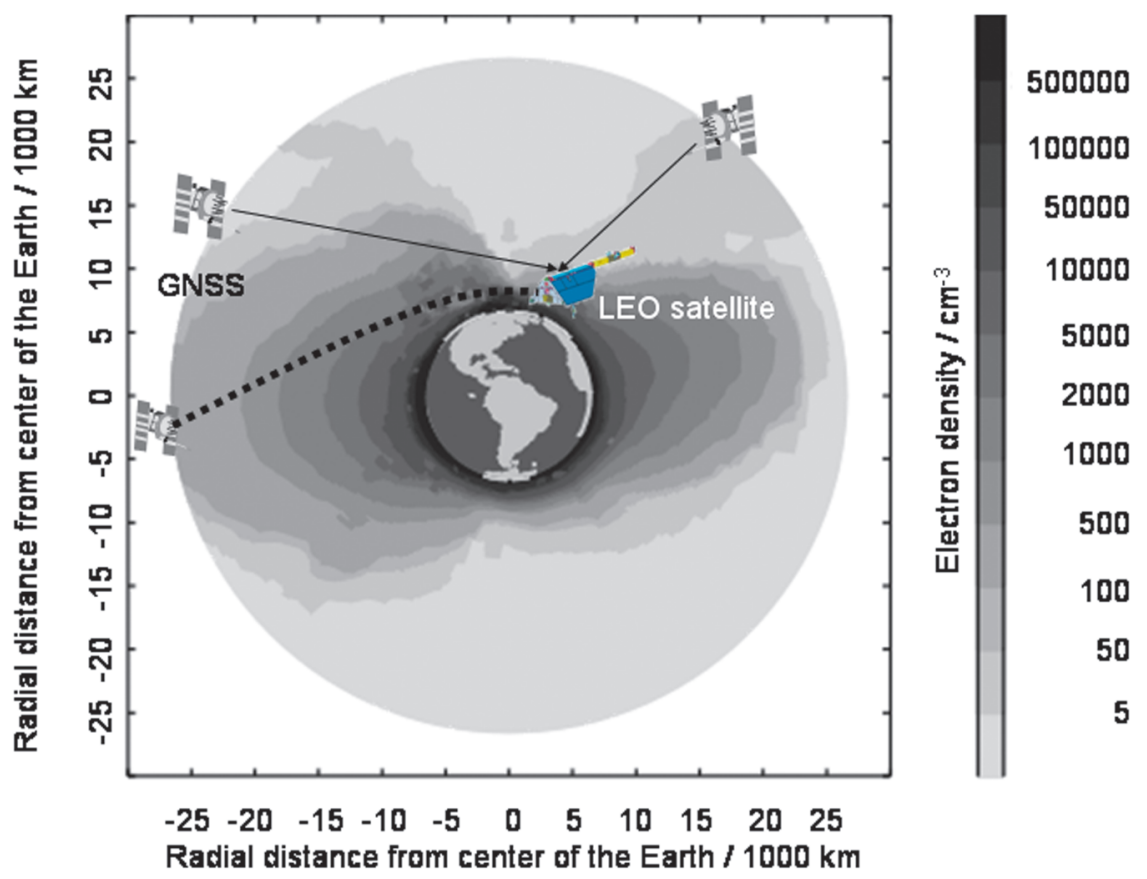

Fig. 3. Schematic view on GNSS based monitoring in SWACI: (1) - ground based GPS monitoring; (2) - radio occultation; (3) - topside reconstruction using GPS navigation data.

In addition to the ground based GPS measurements, measurements onboard Low Earth Orbiting (LEO) satellites such as CHAMP and GRACE are also performed.

Whereas GPS radio occultation measurements provide up to about 150 vertical electron density profiles in anti-velocity direction near the orbit plane (Jakowski, 2005a,b), topside tracking data for satellite positioning are effectively be used to monitor the 3D electron density distribution of the topside ionosphere/plasmasphere near the orbit plane (Heise et al., 2002).

The electron density reconstructions may reveal strong plasma density enhancements in particular in the polar region (Jakowski et al., 2007).

Four types of products/services are delivered to different user groups: warning, nowcast, forecast, post analysis.

\section{Real time corrections using triple frequency techniques}

GNSS dual frequency measurements can be used to reconstruct TEC. Most of the dual frequency TEC reconstruction techniques require the use of code measurements to resolve phase ambiguities. As a consequence, the precision of the reconstructed TEC is affected by code multipath delays and differential satellite and receiver hardware delays (Ciraolo et al., 2007) and is usually limited to 2-3 TEC units at midlatitudes (Warnant and Pottiaux, 2000).

The cornerstone of our approach relies on the use of the third frequency available on Global Navigation Satellite Systems (GNSS), i.e. Galileo and modernized GPS, for an improved TEC reconstruction. As TEC is the key parameter for the mitigation of ionospheric effects on different space based systems, in par- 
ticular on GNSS, a more precise TEC reconstruction would allow to improve the precision and the reliability of many GNSS navigation and positioning techniques.

In our approach, TEC is reconstructed based on undifferentiated triple frequency (L1-L2-L5) GNSS measurements. The basic idea is to form different combinations of triple frequency code and phase measurements in order to solve ambiguities by successive approximations (Spits and Warnant, 2008). This is actually done in three distinct steps.

The objective of the first step is to resolve the so-called extra-widelane (EWL) ambiguities by computing a combination of dual frequency (L2/L5) code and phase measurements. Then the objective of the second step is to resolve the so-called widelane (WL) ambiguities by computing a combination of dual frequency (L1/L2) phase measurements.

In the third step, we introduce the values of those EWL and WL ambiguities in a system of two dual frequency ( $L 1 / L 2$ and $L 2 / L 5)$ phase combinations, which allows us to retrieve the TEC.

In this method, code measurements are only used in the first step, so that code multipath delays and differential satellite and receiver hardware delays do not affect the precision of the TEC. Furthermore, even if phase delays could affect the precision of the reconstructed TEC, we can expect an improvement of one order of magnitude in regards with the dual frequency technique.

As the third frequency was not yet available at the beginning of our work, we have developed software to simulate realistic GPS and Galileo triple frequency code and phase measurements. Thanks to this triple frequency simulation software, we were able to validate our TEC monitoring technique on the three-step level. As far as the first step is concerned, the comparison of the obtained EWL ambiguities with the initial simulated ones shows that it is possible to resolve the EWL ambiguities at their correct integer values. The results of the second step show that as expected we only obtain approximated values of the WL ambiguities. However, it does not prevent us from obtaining the correct TEC values $-i . e$. the values initially simulated - in the third step. In conclusion, the method has been successfully validated on GPS and Galileo simulated data but still requires further validation on real data. We have started to validate our technique on GIOVE-A real data. As far as the first step is concerned, the results on real data are in good agreement with those on simulated data. Further validation is ongoing.

\section{Mitigation techniques in GNSS radio occultation applications}

Phase changes (Doppler shift) in signals from GNSS satellites are the basic measurements of the Radio Occultation (RO) technique (Kursinski et al., 1997). These are caused by the respective motions of the transmitting and receiving satellites, by the Earth's ionosphere, and by the neutral atmosphere (the desired quantity). The kinematic Doppler Effect can be determined and removed via precise knowledge of the satellite's positions and velocities, routinely available from modern precise orbit determination methods. The effect of the ionosphere is frequencydependent (see Section 7) and can therefore be removed to a high degree using a linear combination of measurements at two GNSS frequencies - a process known as «ionospheric correction» (Vorob'ev and Krasil'nikova 1994).

Higher order ionospheric effects, however, are not removed by this approach (see section 7) and ionospheric residual errors are an important part of the error budget in retrieved atmospheric profiles from about $30 \mathrm{~km}$ upwards, where the atmospheric signal is already small (Kursinski et al., 1997). Ionospheric residual errors, in particular systematic ones, are important for RO-based climate monitoring, since potential decadal scale variability of residual ionospheric systematic errors could pretend short-term trends in RO climatologies of the stratosphere (Foelsche et al., 2008). This effect is currently analyzed in detail employing simulation studies over more than two solar cycles.

Climatologies of the upper troposphere and lower stratosphere for the period 2001-2008 (therewith including phases with high and low solar activity) have been built using RO data from the German CHAMP satellite (Foelsche et al., 2007). Comparisons to operational analyses 
from the European Centre for Medium-Range Weather (ECMWF) forecasts show very good overall agreement in the domain below about 30 $\mathrm{km}$ altitude. Differences exceeding $0.5 \mathrm{~K}$ can generally be attributed to ECMWF deficiencies, as they gradually disappear with improvements at ECMWF. Above $30 \mathrm{~km}$ there is a systematic difference between CHAMP and ECMWF, the latter being colder than the former by up to about $2 \mathrm{~K}$ at $35 \mathrm{~km}$ altitude. So far it has not been possible to find any relation between these systematic differences and the phase of the solar cycle. The situation is more favorable when looking at parameters which are closer to the fundamental measurements. Early results using bending angle data at high altitudes (Rocken et al., 2008) indicate a potential way to use information contained in the data to remove ionospheric residual errors. At altitudes above $60 \mathrm{~km}$, where the contribution from the neutral atmosphere is almost negligible, there is a small but systematic difference between measurements during day and night, which is apparently related to the total electron content. Differences between nighttime RO measurements from the COSMIC constellation and climatology for the first four months of 2007 are on average about $-0.4 \cdot 10^{-7} \mathrm{rad}$, while those between day-time measurements and climatology are larger by a factor of $\sim 3$. Initial results based on CHAMP RO data (B. Schreiner, UCAR, Boulder, USA, pers. comm., July 2008) show furthermore, that nighttime differences under low and high solar activity are very similar, while daytime differences show a correlation with solar activity. These results, although preliminary, may show a way to correct for residual ionospheric errors in the context of climate monitoring, where averages over large numbers of RO profiles are used, without relying on external information about the state of the ionosphere. Correcting daytime measurements by the offset between day and night should than remove the major part of ionospheric residuals with variations on decadal scale.

\section{Mitigation of scintillation effects}

The widely used ionospheric scintillation indices $S 4$ and $\sigma_{\varphi}$ represent a practical measure of the intensity of amplitude and phase scintillation affecting GNSS receivers. These indices however do not provide sufficient information regarding the actual tracking errors that degrade GNSS receiver performance when scintillation occurs. Suitable receiver tracking models, sensitive to scintillation (Conker et al., 2003), allow the computation of the variance of the output error of the receiver PLL (Phase Locked Loop) and DLL (Delay Locked Loop), which expresses the quality of the range measurements used by the receiver to calculate user position. The capability of incorporating phase and amplitude scintillation effects into the variance of these tracking errors allows the application of relative weights (based on the inverse of these variances) to measurements from different satellites. This proposed technique gives the least squares stochastic model used for position computation a more realistic representation, in particular under a scintillation scenario, where the ionospheric irregularities will affect each satellite differently. An example of the application of this technique is shown in fig. 4. The plots refer to the error in height when a baseline of $\sim 125 \mathrm{~km}$ in the Arctic region (average geomagnetic latitude $>75^{\circ} \mathrm{N}$ ) is processed on an epoch by epoch relative solution based on $C 1$ and $P 2$ pseudoranges only, during a 24 hour period (10 December 2006) of disturbed geomagnetic conditions $(\mathrm{Kp} \geq 4)$. Results show an overall improvement of about $38 \%$ in height accuracy. Improvement for other baselines, ranging from $1 \mathrm{~km}$ to $750 \mathrm{~km}$, under varying geomagnetic conditions ( $4 \leq \mathrm{Kp} \leq 6)$, varied between 17\% and 34\% (Aquino et al., 2009).

Detailed comparisons with alternative approaches, such as weighting according to satellite elevation angle and by the inverse of the square of the standard deviation of the code/carrier divergence, are presented in (Aquino et al., 2009), where the influence of multipath effects on the proposed mitigation approach is also discussed. Higher levels of $S 4$ may relate to multipath, rather than to actual amplitude fades due to the ionosphere (scintillation), in particular at low elevation angles. The findings in (Aquino et al., 2009) however suggest that with the input of the $S 4$ in the DLL tracking model of (Conker et al., 2003) the pro- 

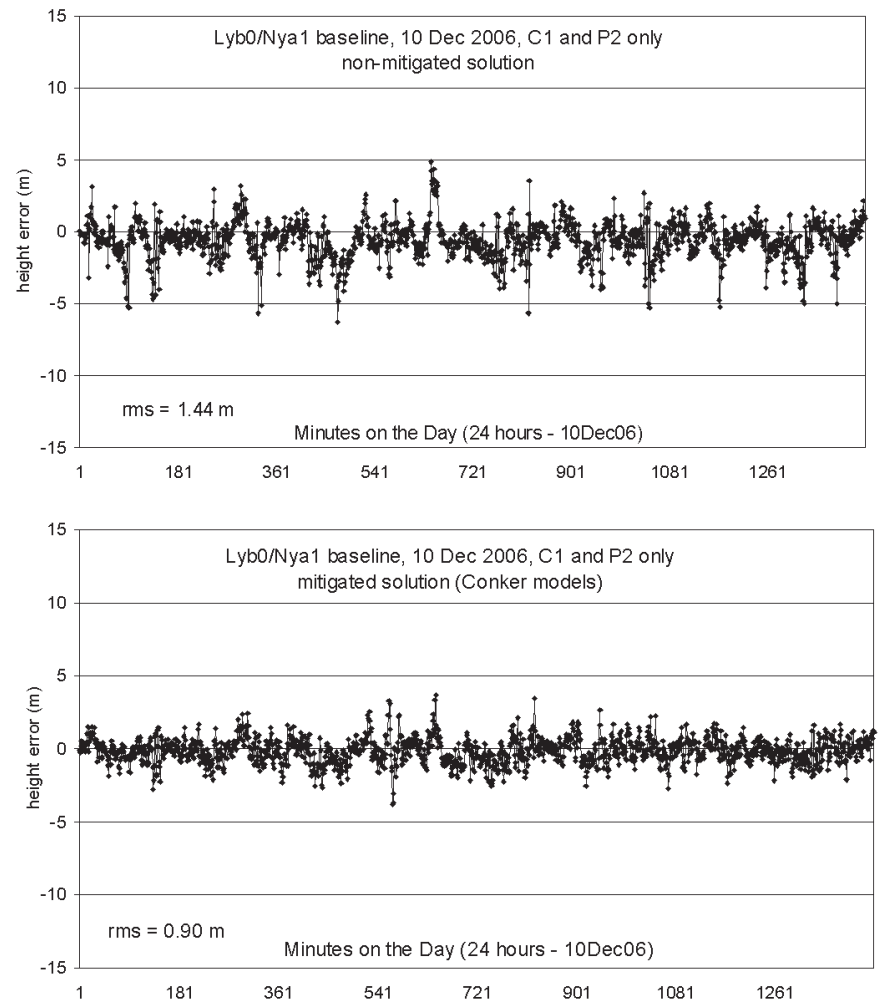

Fig. 4. 24 hours (10 Dec 2006) time series of errors in height for baseline Lyb0/Nya1 when epochwise (1 minute) non-mitigated solution (left below) and mitigated solution (right above) are computed based only on $C 1$ and $P 2$ pseudoranges.

posed approach provides an effective way to improve the solution, also mitigating intervening multipath effects.

To implement the mitigation of the carrier phase solution with the use of the PLL model of (Conker et al., 2003) it is necessary to access the power spectral density (PSD) of fluctuations in the amplitude and carrier phase output, which was possible with the use of high rate scintillation data. Experiments were conducted where a carrier phase-based mitigated solution was also implemented and compared with the conventional solution. During a period of occurrence of high phase scintillation it was observed that problems related to ambiguity resolution can be reduced by the use of the proposed mitigated solution. Details of these results are given in
(Aquino et al., 2008 and 2009). For practical future use of the method in carrier phase positioning it is envisaged that the strategy proposed in (Aquino et al., 2007) and further developed in (Strangeways, 2008) can be applied, where the scintillation indices can be used (in combination with auxiliary high rate phase data) to compute the PLL tracking error variance.

\section{Higher order ionospheric influences in dual frequency systems}

\subsection{Higher order range errors}

Whereas the first-order range error in GNSS applications can be completely eliminated by a 
linear combination of dual frequency measurements at the two frequencies $L 1$ and $L 2$, higher order terms of the refractive index $\mathrm{n}$ given in eq. 7.1 cannot be mitigated in a linear approach.

$$
n \approx 1-\frac{f_{p}^{2}}{2 f^{2}} \pm \frac{f_{p}^{2} f_{g}}{2 f^{3}} \cos \Theta-\frac{f_{p}^{4}}{8 f^{4}}
$$

Here $f$ denotes the signal frequency, $f_{p}$ the plasma frequency $\left(f_{p}<25 \mathrm{MHz}\right), \Theta$ the angle between the Earth's magnetic field vector and the propagation vector and $f_{g}$ the gyro frequency.

Several attempts have been made to mitigate second order effects (e.g. Brunner and Gu, 1991; Bassiri and Hajj, 1993; Jakowski et al., 1994; Hoque and Jakowski, 2006; 2007) applying different approaches. Depending on information on the ray path geometry, the electron density distribution and the shape of the geomagnetic field it is possible to correct the higher order effects with an accuracy of about $1 \mathrm{~mm}$.

Unfortunately, the knowledge of the actual electron density distribution and the geomagnetic field structure is rather poor in operational GNSS applications. Thus, correction formulas taking into account the ionosphere and geomagnetic field structures could be of practical importance. Following this approach, empirical formulas depending only on ray path geometry (azimuth and elevation) and TEC have been developed to compute higher order effects for selected regions with accuracy in the $\mathrm{mm}$ level (Hoque and Jakowski, 2006; 2007). TEC can be provided by real-time ionospheric monitoring services such as SWACI (see Section 3). Due to the anisotropy in the refraction index, introduced by geomagnetic field dependent terms in eq. 7.1, the second order effect depends on the direction of the ray path as it is shown in fig. 5 . Systematic range errors of this type will not cancel out in measurement statistics.

The computation made for a receiver position at $51^{\circ} \mathrm{N}$ and $10^{\circ} \mathrm{E}$, clearly indicates a maximum error in southward direction. Taking into account that the error in the sample shown in fig. 5 can reach up to $60 \mathrm{~mm}$, precise applications require a correction of higher order effects. If more than two frequencies are available as planned in modernized GPS or GALILEO systems, higher order effects can directly be mitigated by using more frequency combinations.

\subsection{Determination and correction of higher order terms for GPS/GNSS rangefinding/positioning}

\subsubsection{Introduction}

Although the standard dual frequency method is able to correct for the majority of the ionosphere induced delay or phase advance, the most accurate positional determination $(1 \mathrm{~cm}$ accuracy or better) requires more precise ionospheric correction, taking also into account the effect of the Earth's geomagnetic field on the refractive index and the curvature of the ray path. However, it is not possible to obtain a precise analytical solution for propagation in an anisotropic media, such as the Earth's ionosphere, because of the non-separable variables in the resulting equations. Although numerical ray-tracing can be employed, the small step size required for $\mathrm{cm}$ accuracy is quite computationally intensive. To overcome this problem, an approximate analytical perturbation method valid at $L$-band has been formulated (Gherm et al., 2006) to calculate the group delay or phase advance for satellite to Earth paths accurately, conveniently and speedily. At these frequencies, utilized for GPS and the future Galileo system, this accuracy was found to be about $2 \mathrm{~mm}$ and comparable with the best previous numerical techniques using high precision ray-tracing (Strangeways and Ioannides, 2002) but with a vastly smaller computational burden.

The method has also been validated against exact analytical calculations for the isotropic case and by the most precise numerical raytracing for the anisotropic case. The method can be used to determine the higher order ionosphere terms due to the geomagnetic field, the square of the electron density along the propagation path (rather than just the TEC) and the curvature of the ray path due to refraction in the ionosphere. Two components of the latter need to be calculated, one due to the gradient perpendicular to the path direction in the plane of propagation and the other perpendicular to the path and the plane of propagation.

This enables the phase advance due to refraction by horizontal gradients to be evaluated 

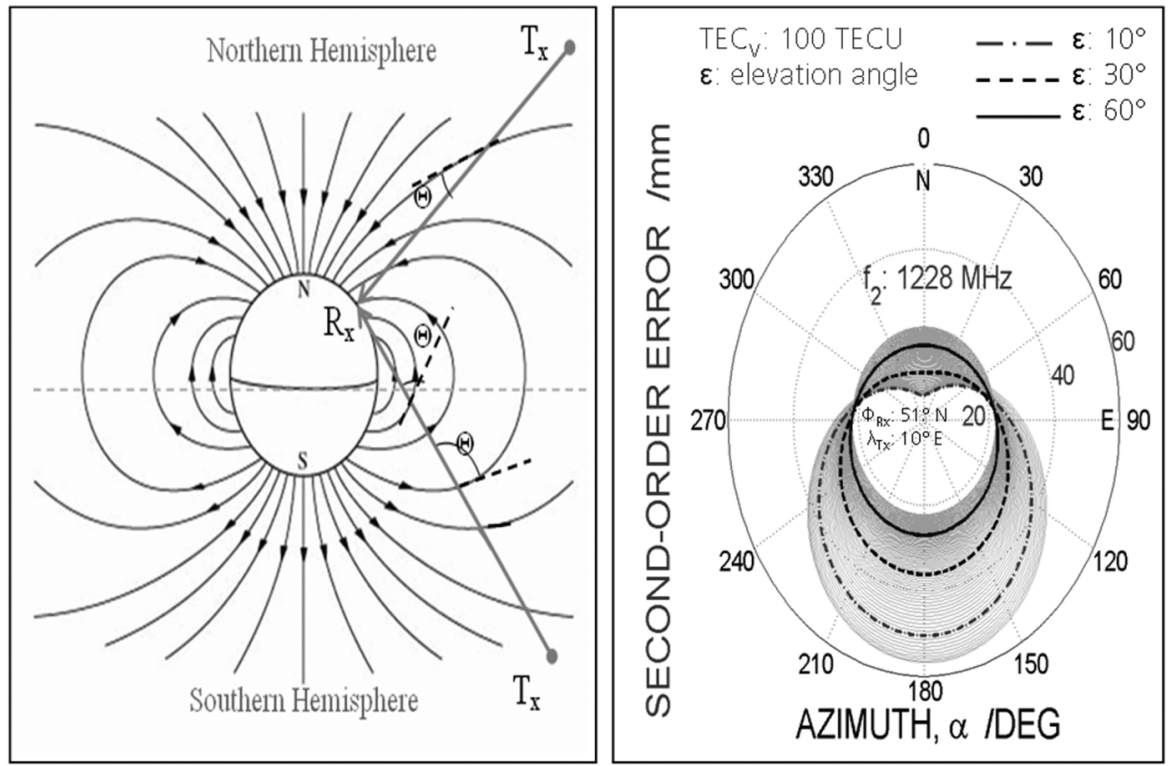

Fig. 5. Left panel: Illustration of the effect of the ray path-magnetic field geometry causing an asymmetry in the refraction. Right panel: Second order ionospheric range error (radial distance from 0-60 mm) for the GPS $L 2$ frequency assuming a vertical TEC of $100 \times 10^{16}$ electrons $/ \mathrm{m}^{2}$ for a mid-European station as a function of azimuth at different elevation angles.

as well as by the vertical electron density gradient.

By determining all the higher order ionosphere errors, the RRE (residual range error) for the GPS or other $L$-band satellite navigation systems using the dual frequency method can also be easily determined. By determining the higher order errors accurately for the conditions pertaining to any GPS satellite to receiver path, these errors can be subtracted from the total phase advance so that only the $f^{-2}$ dependence remains which can be eliminated using the dual frequency method. Although some correction procedures rely on experimental determination of the $f^{-2}$ and $f^{-3}$ terms using 3 frequencies, these will not always be available and, furthermore, the $f^{-3}$ error is very small $(\sim 2$ $\mathrm{cm})$ and with some uncertainty in all the other errors sources, would be very hard to determine accurately in this way.
7.2.2. Description of the perturbation method

The phase advance including the higher order errors terms for ordinary $(0)$ and extra-ordinary $(\mathrm{x})$ modes for a satellite to ground path have been determined using perturbation theory in the geometrical optics equations (Kravtsov and Orlov, 1990) leading to the following formulae:

$$
\begin{aligned}
& L_{\text {mes }}^{0, e}(\text { in metres })=L_{\text {real }}-\frac{40,311}{f^{2}} \\
& \int_{0}^{L} N(s) d s \pm \frac{40.311}{f^{3}} \int_{r 0}^{L}\left(f_{H} \cos \Psi\right) N(s) \\
& d s-\left(\frac{812.48}{f^{4}} \int_{0}^{L} N^{2}(s) d s+\frac{1}{2} \int_{0}^{L} \mathbf{1}_{1}^{2}(s) d s\right) \\
& \mathbf{1}_{1}(s)=\mathbf{1}_{1}^{0}-\frac{1}{2} \int_{0}^{L}(1 \pm Y \cos \Psi) \nabla_{\perp} X(r(s)) d s
\end{aligned}
$$




$$
\begin{aligned}
& \mathbf{l}_{1}^{0}=\mp \frac{1}{2 L} \int_{0}^{L} X Y \mathbf{h}_{\perp} d s+\frac{1}{2 L} \int_{0}^{L} d s \\
& \int_{0}^{s}(1 \pm Y \cos \Psi) \nabla_{\perp} X(r(s)) d s
\end{aligned}
$$

The equations (7.2 to 7.4 ) have been verified and validated by comparison with the rigorous solution of the problem for the isotropic spherically layered ionosphere. They have also been validated for the more general case of an arbitrary 3D ionosphere with horizontal ionospheric gradients and permeated geomagnetic field (Gherm et al., 2006) by comparison with the most precise ray-tracing calculations (Strangeways and Ioannides, 2002).

\subsubsection{Calculation of the higher order errors on GPS}

The program enables the main error due to the electron density (proportional to $f^{-2}$ ) as well as the higher order terms in $f^{-3}$ and $f^{-4}$ to be determined (Strangeways et al., 2007a). An RRE (Residual Range Error) will result from application of the standard dual frequency model due to the incorrect weighting of these terms (assumed $f^{-2}$ rather than $f^{-3}$ or $f^{-4}$ dependence) and this can also be determined. An example of such a calculation for the $L 1$ GPS frequency is given in fig. 6. This shows all these errors for a profile with a vertical TEC of 72.6 for the extraordinary mode determined for a GPS satellite to ground $\left(20^{\circ}\right.$ latitude) path at zero azimuth to the magnetic meridian. A value of the horizontal electron density gradient, normalised to the local electron density, of $0.001 / \mathrm{km}$ was employed at all heights. A recent extension to the program permits actual horizontal gradients to be determined using the 3D NeQuick ionosphere model and incorporates the realistic $\mathrm{CO}_{2}$ geomagnetic field model (Strangeways et al., 2007b). The distance errors due to the relative contributions to the phase advance can be seen in the figure for both the curvature in the plane of propagation and perpendicular to it. The doted line, representing the higher order term due to the square of the profile of electron density along the path is the smallest error term for elevations less than $30^{\circ}$ for these parameters. The geomagnetic field term is generally the largest of these error sources at $L$-band, although not for very low elevation paths. The direction of the error due to the magnetic field is reversed if the direction of the path is North to South rather than South to North or for the other magneto-ionic mode. Consequently, sometimes the geomagnetic field induced and curvature induced errors will add and sometimes cancel each other. The RRE for dual frequency $L 1 / L 2$ is also plotted taking account of the effect all the higher error terms would have on this determination.

\subsubsection{Discussion concerning determination of the higher order errors.}

The second order error term involves an integral along the ray path of the product of the electron density and the component of the geomagnetic field in the direction of the wave normal. Some workers find an approximation of this using the TEC multiplied by $B$ at some particular altitude along the path which takes into account the «weighting» of the product with the actual (varying) electron density along the path. This altitude tends to be higher than the electron density maximum and also shows a latitudinal dependence. Others use the ionosphere shell approximation and determine the product at the pierce point at the shell altitude. The latter is less accurate. However, calculation of this term by integration of this product along the path shows that it can take two distinct forms which depend on geomagnetic latitude and path elevation (Gherm et al., 2006). Thus care must be taken using the approximate treatments mentioned above.

The third order error terms exist due to terms in $f^{-4}$ in the expansion for the refractive index which yield corresponding terms in both phase advance and group delay (of which only the first is normally considered) and also due to refraction (curvature) of the ray path. This takes place both in the plane of propagation and perpendicular to it. It is important to note that the curvature term cannot be determined by the additional length of the refracted path compared with the l.o.s. (line of sight path) as the refraction of the path results in propagation in regions 


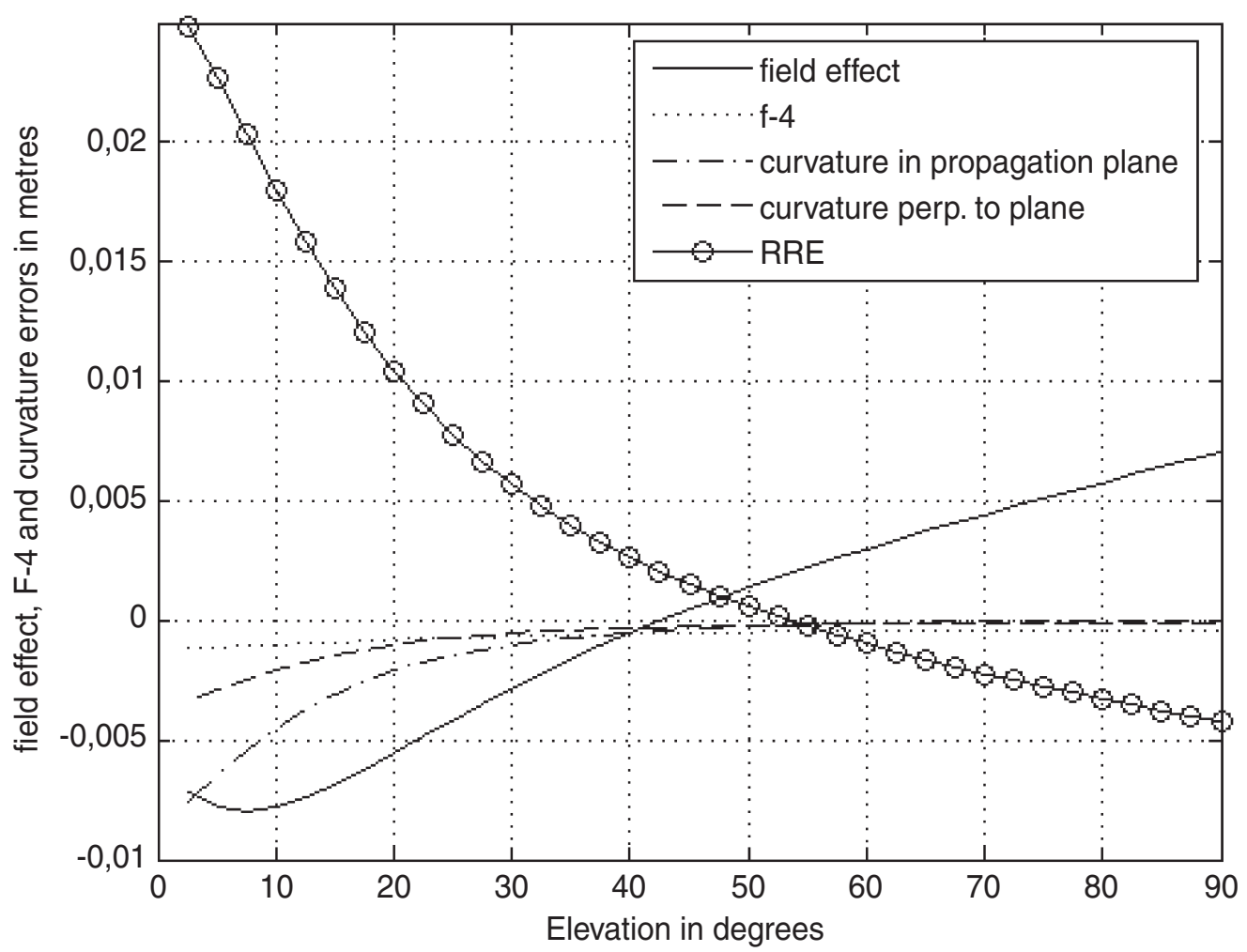

Fig. 6. Higher order errors for the GPS L1 frequency due to the $f^{-3}$ and $f^{-4}$ terms (including curvature terms) and the resultant RRE for dual frequency $L 1 / L 2$.

where the refractive index is different from that for the 1.o.s path (Strangeways, 2008). For the phase path, this difference in refractive index results in a phase advance which is generally about double the magnitude of the phase delay caused by the additional path length but in the opposite direction.

Thus the refraction causes the phase path to be more advanced than it would be for the l.o.s and the error is of opposite sign to that which would be determined on the basis of just the difference in path length.

\subsubsection{Discussion of correction procedures}

Employing the standard dual frequency scheme, the approximate slant TECs for the paths to the receiver from all the satellites visible at any one time can be determined. Then the electron density profiles used to determine the higher order error terms can be normalised to these TEC values. Profiles can be chosen from an ionosphere model such as IRI for the appropriate time of day, month, sunspot number etc. The magnitude of the higher order terms for each satellite to receiver path, using their known azimuth and elevation (based on an approximate receiver location) and an assumed geomagnetic field model, can then be determined. Then the higher order terms for each path can be subtracted from the observed values of $L 1$ and $L 2$ to determine corrected ranges for all the satellites. For phase this will of course also involve resolving the ambiguities. The most accurate estimate of the second order er- 
ror can be found by integration along the whole path using the formula given in eq. 7.2. Such a correction strategy was employed on simulated data by Nagarajoo et al. (2007) and this error found to be reduced by about one order of magnitude (e.g from $1.7 \mathrm{~cm}$ to $1.7 \mathrm{~mm}$ ).

The curvature error can also be calculated using the perturbation formulae given above (the last term in eq. 7.2 using also eq. 7.3 and 7.4). This term depends strongly on the electron density gradients at all points along the path and these then need to be known in both the plane of propagation and in the direction perpendicular to it. In our program these are given using the NeQuick 3D ionosphere model. Since the gradients from this model will only be an approximation to the real case, the percentage error in predicting this term is larger than for the second order term. However the curvature term is generally about an order of magnitude smaller than the second order term at GPS frequencies, except for low elevations that are unlikely to be used for ground-observed GPS satellites. Then, the absolute error in determining the curvature term is still likely to be smaller than that in the determination of the second order (geomagnetic field) error. Kim and Tinin (2007) have also independently obtained a formula for the third order term using perturbation theory. Their formulation also includes the effect of ionosphere irregularities which the present authors have treated separately (Gherm et al., 2005).

\section{Effects of local structures in the iono- sphere on real time high precision GNSS applications}

Nowadays, Global Navigation Satellite Systems or GNSS measure positions in real time with an accuracy ranging from a few meters to a few centimeters. The best precisions can be reached in differential mode using phase measurements. In differential mode, mobile users improve their positioning precision thanks to so-called «differential corrections» provided by a reference station. For example, the Real Time Kinematic technique (RTK) measures positions in real time with a preci- sion usually better than a decimeter when the distance between the reference station and the user is smaller than $20 \mathrm{~km}$. In practice, the ionospheric effects on GNSS radio signals remain the main factor which limits the precision and the reliability of differential positioning. As differential applications are based on the assumption that the measurements made by the reference station and by the mobile user are affected in the same way by the ionospheric effects, these applications are affected by gradients in TEC between the reference station and the user. For this reason, local variability in the ionospheric plasma can be the origin of strong degradations in positioning precision. GNSS real time users who undergo degradations in their applications are not necessarily aware of this problem: this is an important limitation to the reliability of GNSS positions. For this reason, we decided to characterize ionospheric variability which can pose a threat to the RTK positioning technique.

As already mentioned, small-scale variability in the TEC is the origin of degraded GNSS positioning conditions. Therefore, in a first step, we performed a detailed study of the main ionospheric phenomena which can induce local variability in TEC. In practice, GNSS carrier phase measurements can be used to monitor local TEC variability: small-scale ionospheric structures can be detected by monitoring TEC high frequency changes at a single station; as ionospheric disturbances are moving, we can expect that such structures will induce TEC temporal variability which can be detected at a single station (Warnant and Pottiaux, 2000). We applied this method (called «one-station» method) to the GPS data collected at the permanent (mid-latitude) station of Brussels from 1994 to 2006. Two main types of structures have been observed: Travelling Ionospheric Disturbances (TID's) and «noise-like» structures. We call «noise-like structures», the variability in TEC shown in fig. 7. We analysed the maximum daily Rate of TEC (RoTEC) values observed at Brussels between January 2001 and December 2006. We have afterwards grouped these values according to the seasons: we obtained the maximum seasonal RoTEC values which appear in the table II. 
Table II. Maximum seasonal RoTEC values at Brussels from January 2001 to December 2006 (expressed in $\mathrm{TECU} / \mathrm{min})$.

\begin{tabular}{lcccc}
\hline \hline & Spring & Summer & Autumn & Winter \\
\hline 2001 & 6.881 & 1.122 & 4.028 & 9.068 \\
2002 & 0.745 & 1.821 & 1.946 & 2.211 \\
2003 & 0.693 & 0.653 & 9.839 & 1.231 \\
2004 & 1.581 & 1.152 & 0.861 & 1.263 \\
2005 & 1.234 & 2.579 & 0.503 & 1.276 \\
2006 & 0.582 & 1.197 & 0.805 & 0.845 \\
\hline
\end{tabular}

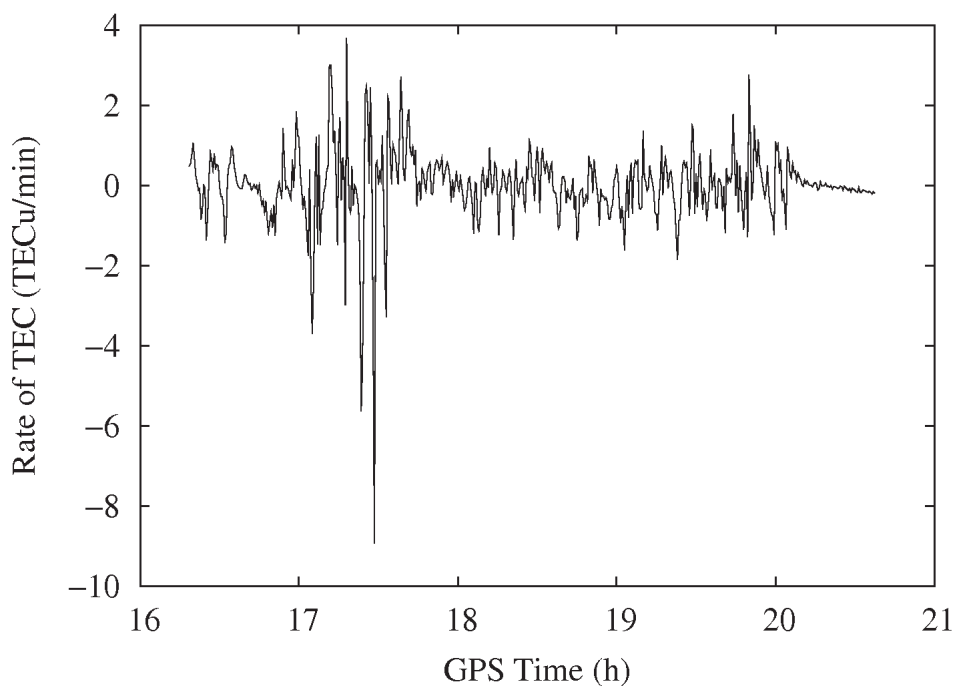

Fig. 7. Ionospheric variability at Brussels on 20th November 2003 (satellite 2).

The largest RoTeC detected at Brussels were observed during severe geomagnetic storms. For example, the storm of the DOY 303/03 (30th October 2003) which presented a DST minimum index of $-383 \mathrm{nT}$ was responsible for the largest RoTEC gradients observed during the period 1994-2006: 9.839 TECU/min. RoTEC due to TID's are much smaller than RoTEC due to geomagnetic storms: the analysis of a lot of TID cases shows that the maximum RoTEC value observed during the occurrence of a TID was about $1.5 \mathrm{TECU} / \mathrm{min}$. Moreover, the analysis of table II shows that strong irregularities occur even during solar minimum, for example, during the summer 2006, where gradients up to 1.2 TECU/min were reached. In addition, let us underline that this maximum RoTEC of summer 2006 was larger than the maximum RoTEC of summer 2001 (solar maximum). This means that, even during periods where the probability of occurrence of ionospheric irregularities is very low, large RoTEC can be observed. This is 
a very important issue for GNSS users.

The one-station method allows to measure TEC variability in time but GNSS differential applications are affected by variability in space between the user and the reference station. Therefore, in a second step, we developed software which reproduces, as far as possible, positioning conditions experienced by RTK users on the field (Lejeune and Warnant, 2008). We used this software to assess ionospheric effects on RTK for 4 days representative of different «typical» ionospheric conditions: quiet ionospheric activity (as measured by the one-station method), medium and large amplitude TID's and noise-like variability due to a severe geomagnetic storm (20th November 2003). We considered the case of users who have already fixed their phase ambiguities to the correct integer. Again, the largest effects were observed during the geomagnetic storm of 20th November 2003 where the positioning error due to ionosphere reached $80 \mathrm{~cm}$. The maximal values of this error observed for TID's were $15 \mathrm{~cm}$ and $22 \mathrm{~cm}$, respectively for the medium-amplitude TID and the large-amplitude TID. During quiet days in terms of ionospheric variability, the positioning error due to ionosphere is typically $1-2 \mathrm{~cm}$ but reached $5 \mathrm{~cm}$, which is still within the usual RTK accuracy level. We have also studied the case where ionospheric disturbances occur during the ambiguity resolution process: as soon as the ionospheric residual error reaches $10 \mathrm{~cm}$ (a half wavelength), the ambiguity resolution process can be corrupted and positioning errors are growing up to several meters. During our study, we also found that there is a relationship between the baseline length and the effects on positioning: positioning error due to ionosphere increases with increasing baseline length. In addition, for a given ionospheric disturbance, the positioning error due to the ionosphere depends very much on baseline orientation.

\section{Conclusions}

The paper reviews work performed in the frame of Work Package 3.2 «Mitigation techniques»of COST Action 296 to improve ionosphere mitigation techniques in the frame of different applications and to assess their remaining weaknesses. As the way the ionosphere influences GNSS data processing techniques depends very much on the type of application, specific mitigation techniques have to be developed.

At the present time, a growing number of users need results in real time. Such applications are particularly vulnerable to ionospheric threats. Therefore, we put the focus on real time ionospheric corrections. The Galileo Single Frequency Ionospheric Correction Algorithm is based on the NeQuick model; remaining weaknesses in this model have been investigated at the University of Liege mainly by comparing NeQuick TEC with GPS TEC. Ground and Space based GNSS observations provide a continuous flow of information that can be used for real time monitoring of the characteristics of the ionosphere and its dynamic processes. Finnish Meteorological Institute has assessed the feasibility of using observations from a regional GNSS network in Finland to perform mesoscale ionospheric tomography in the Auroral region. Deutsches Zentrum für Luft- und Raumfahrt (DLR) Neustrelitz has established a near real time ionospheric data service using ground and Space based GNSS measurements. At the Royal Meteorological Institute of Belgium, a new TEC reconstruction technique is being developed based on triple frequency Galileo or (modernized) GPS measurements. The increased accuracy in TEC reconstruction will allow to improve ionosphere mitigation techniques used in many applications. The University of Graz has investigated ionosphere mitigation techniques used in the frame of radio occultation experiments of which the goal is to retrieve neutral atmosphere parameters. The University of Nottingham has developed a technique allowing to mitigate scintillation effects on GNSS positioning.

Many GNSS applications rely on the dual frequency correction for the mitigation of ionospheric effects; the remaining influence of higher order terms has been investigated at DLR, University of Leeds and University of St. Petersburg.

Local variability in TEC can be the origin of strong degradation in high precision real time 
positioning applications. The Royal Meteorological Institute of Belgium and the Geophysical Institute of the Bulgarian Academy of Sciences have characterized small-scale structures in TEC which can pose a threat for these applications.

\section{Acknowledgements}

The investigations presented here were mainly carried out and coordinated in the frame of the COST 296 activity «Mitigation of Ionospheric Effects on Radio Systems (MIERS)». Work package leaders thank very kindly all contributors to this very challenging scientific topic.

\section{REFERENCES}

Aquino, M., M. Andreotti, A. Dodson and H.J. STRAngeWAIS (2007): On the Use of Ionospheric Scintillation Indices as Input to Receiver Tracking Models, $A d$ vances in Space Research, 40 (3), 426-435.

Aquino, M., J.F.G. Monico, H.G. Marques, A. Dodson, G. De Franceschi, L. Alfonsi, V. Romano and M. ANDREOTTI (2008): Using Ionospheric Scintillation Indices to Study Receiver Tracking Performance and Mitigate Positioning Errors, in Workshop on Ionospheric Scintillation: Scientific Aspects, Space Weather Application and Services, 20-22 February 2008, Nottingham, UK.

Aquino, M., J.F.G. Monico, A. Dodson, H.G. Marques, G. De Franceschi, L. Alfonsi, V. Romano and M. ANDREOTTI (2009): Improving the GNSS Positioning Stochastic Model in the Presence of Ionospheric Scintillation, Journal of Geodesy, doi:10.1007/s00190-009-0316-6.

Austen, J., S. Franke and C. LiU (1988): Ionospheric imaging using computerized tomography, Radio Sci., 23 (3), 299-307.

BASSIRI, S. and G.A. HAJJ (1993): Higher-order Ionospheric Effects on the Global Positioning System Observables and Means of Modeling Them, Manuscripta Geodaetica, 18, 280-289.

BIDAINE, B. and R. WARNANT (2007): Assessment of the NeQuick Model at Mid-latitudes Using GPS TEC and Ionosonde Data, Proc. 1st Colloquium Scientific and Fundamental Aspects of the Galileo Programme.

BRUNNER, F.K. and M. Gu (1991): An Improved Model for the Dual Frequency Ionospheric Correction of GPS Observation, Manuscripta Geodaetica, 16, 205-214.

Ciraolo, L., F. Azpilicueta, C. Brunini, A. Meza and S.M. RADICELLA (2007): Calibration errors on experimental slant total electron content (TEC) determined with GPS, Journal of Geodesy, 81 (2), 111-120.

Conker, R.S., M.B. El-Arini, C.J. Hegarty and T. Hsiao
(2003): Modelling the Effects of Ionospheric Scintillation on GPS/Satellite-Based Augmentation System Availability, Radio Science, 38, 1001, doi:10.1029/2000RS002604.

Foelsche, U., M. Borsche, A.K. Steiner, A. Gobiet, B. Pirscher, G. Kirchengst, J. Wickert and T. SChMidT (2007): Observing Upper Troposphere-Lower Stratosphere Climate with Radio Occultation Data from the CHAMP Satellite, Climate Dynamics, 31, 49-65, doi:10.1007/s00382-007-0337-7.

Foelsche, U., G. Kirchengst, A.K. Steiner, L. KornBLUEH, E. MANZinI and L. BengtsSON (2008): An observing system simulation experiment for climate monitoring with GNSS radio occultation data: setup and testbed study, J. Geophys. Res., 113, D11108, doi:10.1029/2007JD009231.

Fremouw, E., J. Secan and B. Howe (1992): Application of stochastic inverse theory to ionospheric tomography, Radio Sci., 27 (5), 721-732.

GHeRM, V.E., N.N. ZeRnov and H.J. STRANGeways (2005): Propagation model for transionospheric fluctuating paths of propagation in a wideband ionospheric fluctuating reflection channel: Physically based software simulator of the transionospheric channel, Radio Science, 40 (1), RS1003, doi: 10.1029/2004RS003097.

GHERM, V.E., R. NOVITSKY, N.N. ZERNOv, H.J. STRANGEWAYS and R.T. IOANNIDES (2006): On the limiting accuracy of range measurements for the three-frequency mode of operation of a satellite navigation system, COST 296 workshop, Rennes, France, 4-6 October 2006.

Heise, S., N. Jakowski, A. WehrenpfenNig, Ch. Reigber and H. LÜHR (2002): Sounding of the Topside Ionosphere/Plasmasphere Based on GPS Measurements from CHAMP: Initial Results, Geophys. Res. Lett., 29, 14, doi:10.1029/2002GL014738.

HoQUE, M.M. and N. JAKOWSKI (2006): Higher order ionospheric effects in precise GNSS positioning, J. Geodesy, 81, 259-268, doi:10.1007/s00190-006-0106-0.

HoQue, M.M. and N. JAKOwSKI (2007): Mitigation of higher order ionospheric effects on GNSS users in Europe, GPS Solutions, 12, 87-97, doi:10.1007/s10291-0070069-5.

JAKOWSKI, N., F. Porsch and G. MAYER (1994): Ionosphere-Induced-Ray-Path Bending Effects in Precise Satellite Positioning Systems, Zeitschrift fuer Satellitengestuetzte Positionierung, Navigation und Kommunikation, 3, 6-13.

JAKOWSKI, N. (2005a): Radio Occultation Techniques for Probing the Ionosphere, Radio Science Bull., 314, 4-15.

JAKOWSKI, N. (2005b): Ionospheric GPS Radio Occultation measurements on board CHAMP, GPS Solutions, 9, 88-95, doi:10.1007/s10291-005-0137-7.

Jakowski, N., V. Wilken, S. Schlueter, S.M. Stankov and S. HEISE (2005a): Ionospheric space weather effects monitored by simultaneous ground and space based GNSS signals, J. Atmos. Solar-Terr. Phys., 67, 1074-1084.

Jakowski, N., S. Stankov, D. Klaehn, J. RuefFer, B. HuCK and A. RIETDORF (2005b): Ionospheric impact on the performance of GNSS reference networks, CDROM, Proc. The European Navigation Conference, GNSS 2005, 19-22 Juli 2005, Munich, Reg._Nr.: 2025292005.

JAKOWSKI, N., S.M. StanKov and D. KLAEHN (2005c) Op- 
erational space weather service for GNSS precise positioning, Ann. Geophys., 23, 3071-3079.

Jakowski, N., H. MaAss, S.M. Stankov, K.D. Missling, C. BeCKer, C. Mayer, M.M. HoQue, V. RudenKo and M. TEGLER (2006): SWACI - Space weather service for high precision GNSS positioning, the 3rd European Space Weather Week, (Brussels, 13-17 November 2006).

JAKOWSKI, N., V. WILKEN and C. MAYER (2007): Space weather monitoring by GPS measurements on board CHAMP, Space Weather, 5, S08006, doi:10.1029/2006SW000271.

KIM, B.C and M.V. TININ (2007): Contribution of ionospheric irregularities to the error of dual frequency positioning, J. Geodesy, 81, 186-199, doi:10.1007/s00190006-0099-8.

Kravtsov, Yu. and Yu. ORLOV (1990): Geometrical Optics of the Inhomogeneous Media, (Springer-Verlag, Berlin, Heidelberg).

KuRsinski, E.R., G.A. HaJJ, J.T. Schofield, R.P. LiNFIELD and K.R. HARKY (1997): Observing Earth's atmosphere with radio occultation measurements using GPS, J. Geophys. Res., 102, 23429-23465.

Lee, J.K., F. Kamalabadi and J.J. MaKela (2007): Localized three-dimensional ionospheric tomography with GPS ground receiver measurements, Radio Sci., 42, RS4018, doi:10.1029/2006RS003543.

LEJEUNE, S. and R. WARNANT (2008): A novel method for the quantitative assessment of the ionosphere effect on high accuracy GNSS applications which require ambiguity resolution, J. of Atmospheric and Solar-Terrestrial Physics, 70, 889-900, doi:10.1016/j.jastp.2007.01.009.

Ma, X.F., T. Maruyama, G. Ma and T. Takeda (2005): Three-dimensional ionospheric tomography using observation data of GPS ground receivers and ionosonde by neural network, J. Geophys. Res., 110, A05308, doi:10.1029/2004JA010797.

Materassi, M. and C.N. Mitchell (2005): Imaging of the equatorial ionosphere, Ann. Geophys., 48 (3), 477 482.

Meggs, R.W., C.N. Mitchell and P.S.J. SPencer (2004): A comparison of techniques for mapping total electron content over Europe using GPS signals, Radio Sci., 39, RS1S10, doi:10.1029/2002RS002846.

Meggs, R.W., C.N. Mitchell and V.S.C. Howells (2005): Simultaneous observations of the main trough using GPS imaging and the EISCAT radar, Ann. Geophys., 23 (3), 753-757.

Mitchell, C.N. and P.S.J. SPENCER (2003): A three-dimensional time-dependent algorithm for ionospheric imaging using GPS, Ann. Geophys., 46, 687-696.

Nagarajoo, K., H.J. Strangeways and R.T. IoAnnides (2007): Improving the dual frequency ionospheric correction in GPS positioning by taking account the higher order terms, paper presented at the 20th UK URSI Colloquium at Portsmouth University, 2-3 July 2007.

NAva, B., P. Coisson and S.M. Radicella (2008): A new version of the NeQuick ionosphere electron density model, J. Atmos. and Sol.-Terr. Phys., 70, 1856-1862, doi:10.1016/j.jastp.2008.01.015

Orus, R., B. Arbesser-Ratsburg, R. Prieto-Cerdeira, M. Hernandez-Pajares, J.M. Juan and J. SANZ (2007):
Performance of Different Ionospheric Models for Single Frequency Navigation Receivers, Proc. Beacon Satellite Symposium 2007.

Radicella, S.M. and R. Leitinger (2001): The evolution of the DGR approach to model electron density profiles, Adv. Space Res., 27 (1), 35-40. http://dx.doi.org/10.1016/S0273-1177(00)00138-1.

Rocken, C., S. Sokolovskiy, B. Schreiner, D. Hunt, B. Ho, B. Kuo and U. Foelsche (2008): Climate Monitoring with Radio Occultation Data - Systematic Error Sources, Workshop on the Applications of GPS Radio Occultation to Climate, (Boulder, Colorado, USA, 1718 March, 2008).

SPITS, J. and R. WARNANT (2008): Total Electron Content monitoring using triple frequency GNSS data: a three-step approach, Journal of Atmospheric and Solar-Terrestrial Physics, $\mathbf{7 0}$ (15), 1885-1893, doi:10.1016/j.jastp.2008.03.007.

STRANGEWAYS, H.J. and R.T. IOANNIDES (2002): Rigorous calculation of ionospheric effects on GPS Earth-satellite paths, Acta Geodaetica et Geophysica, 2-3, 281-292.

StRANGEWAYs, H.J., V.E. GHERM and N.N. ZERNOV (2007a): A new fast convenient method of determining ionospheric higher order error terms for dual or triple frequency correction methods, International Beacon Satellite Symposium, (Boston, USA, 11-15 June 2007).

Strangeways, H.J., V.E. GHerm and N.N. Zernov (2007b): Fast determination of path delays and ray path curvature between satellites through a $3 \mathrm{D}$ ionosphere for L-band transmissions, presented at the European Conference on Antennas and Propagation Edinburgh, (Nov 11-16, 2007, published on conference CD-ROM).

StRANGEWAYS, H.J. (2008): Determining scintillation effects on GPS receivers, Ionospheric Effects Symposium IES2008, (Alexandria, Virginia, USA, 13-15 May 2008).

STRANGEWAYS, H.J. (2008): Determination and correction of higher order ionosphere error terms in GPS rangefinding, presented at the XXIXth General Assembly of URSI, (Chicago, Illinois, USA, 7-16 August 2008).

VoroB'EV, V.V. and T.G. KrASIL'NIKOVA (1994): Estimation of the accuracy of the atmospheric refractive index recovery from Doppler shift measurements at frequencies used in the NAVSTAR system, Phys. Atmos. Ocean, 29, 602-609.

WARnant, R. and E. PotTiaux (2000): The increase of the ionospheric activity as measured by GPS, Earth, Planets and Space, 52 (11), 1055-1060.

Wen, D., Y. Yuan, J. OU, K. Zhang and K. Liu (2008): A Hybrid Reconstruction Algorithm for 3-D Ionospheric Tomography, Geoscience and Remote Sensing, IEEE Transactions on Geoscience and Remote Sensing, 46 (6), 1733-1739, doi:10.1109/TGRS.2008.916466.

Yin, P., C.N. MitChell, P.S.J. SPENCER and J.C. Foster (2004): Ionospheric electron concentration imaging using GPS over the USA during the storm of July 2000, Geophys. Res. Lett., 31, L12806, doi:10.1029/2004GL019899.

YIN, P. and C.N. MitchelL (2005): Use of radio-occultation data for ionospheric imaging during the April 2002 disturbances, GPS Solutions, 9 (2), 156-163. 